\title{
Nanostructuring of Transparent Materials by Light
}

\author{
Peter G. Kazansky ${ }^{1,3}$, Yasuhiko Shimotsuma ${ }^{2}$, Jianrong Qiu ${ }^{1}$ \& Kazuyuki Hirao ${ }^{4}$ \\ 'Photon Craft Project, Japan Science and Technology Corporation and Shanghai Institute of Optics and Fine Mechanics, \\ Chinese Academy of Sciences, Keihanna-Plaza, Kyoto 619-0237, Japan \\ ${ }^{2} R \& D$ center Kagoshima, Kyocera Corporation, Kagoshima, Kokubu 899-4312, Japan \\ ${ }^{3}$ Optoelectronics Research Centre, University of Southampton, SO17 1BJ, United Kingdom \\ ${ }^{4}$ Department of Material Chemistry, Graduate School of Engineering, Kyoto University, Kyoto, Sakyo-ku 606-8501, Japan
}

The smallest embedded structures ever created by light are observed in the experiments on femtosecond direct writing. The phenomenon is interpreted in terms of interference between the incident light field and the electric field of bulk electron acoustic wave, resulting in periodic structural changes in glass.

Progress in high power ultra-short pulse lasers has opened new frontiers in physics and technology of lightmatter interactions from coherent $\mathrm{x}$-ray generation to 3D direct writing of photonic structures [1-4]. A critical advantage of using femtosecond pulses relative to longer pulses for optical writing and data storage is that such pulses can rapidly and precisely deposit energy in solids [5, 6]. However, fabrication of sub-wavelength structures by light still is a challenging problem. Surface gratings with a period equal to the wavelength of incident light have been observed in many experiments involving laser deposition and laser ablation [7]. Here we report the first observation of periodic structures within the bulk of a transparent material written by a single femtosecond infrared laser beam. The structures in silica glass consist of oxygen depleted regions of $20 \mathrm{~nm}$ size with periods as small as $140 \mathrm{~nm}$. These are the smallest embedded structures ever created by light.

Although molecular defects caused by intense femtosecond irradiation have been identified in fluorescence, ESR and other studies, the mechanism of induced material modifications is still not fully understood $[8,9]$. Recent observations of anisotropic light scattering and reflection from the regions modified by intense femtosecond light pulses have given the evidence of sub-wavelength index gratings imprinted in irradiated materials $[10,11]$. Form birefringence induced by self-organized sub-wavelength index gratings has been also proposed to explain a puzzling phenomenon of uniaxial birefringence of structures written within fused silica plates $[11,12]$. However, until now there has been no direct proof of existence of such gratings.

In our experiments we used commercially available synthetic silica glass samples. The laser radiation produced by regenerative amplified mode-locked Ti:Sapphire laser (150 fs pulse duration, $200 \mathrm{kHz}$ repetition rate) operating at a wavelength of $800 \mathrm{~nm}$ was focused via $100 \mathrm{x}(\mathrm{NA}=0.95)$ microscope objective into the silica glass samples.

After laser irradiation the sample was polished to the depth of the beam waist location. The surface of the polished sample was analyzed by scanning electron microscope and Auger electron spectroscopy. Secondary electron (SE) images and backscattering electron (BE) images of the same surface were compared (Fig. 1). The SE images of the polished silica sample indicate that the morphology of an irradiated sample in the examined cross-section does not change, namely, a void does not exist. On the other hand, the $\mathrm{BE}$ images reveal a periodic structure of stripe-like dark regions with low density of material and of $\sim 20 \mathrm{~nm}$ width which are aligned perpendicular to the writing laser polarization direction. The Auger spectra mapping indicates that the oxygen defects $\left(\mathrm{SiO}_{2-\mathrm{x}}, \mathrm{x} \sim 0.4\right)$ are periodically distributed in the focal spot of the irradiated region.

The grating periods were about $240 \mathrm{~nm}, 180 \mathrm{~nm}$ and 140 $\mathrm{nm}$ for the number of light pulses of $5 \times 10^{4}, 20 \times 10^{4}$ and 80 $\times 10^{4}$ respectively and for the pulse energy of $1 \mu \mathrm{J}$. Grating periods of $180 \mathrm{~nm}, 240 \mathrm{~nm}$ and $320 \mathrm{~nm}$ were measured at
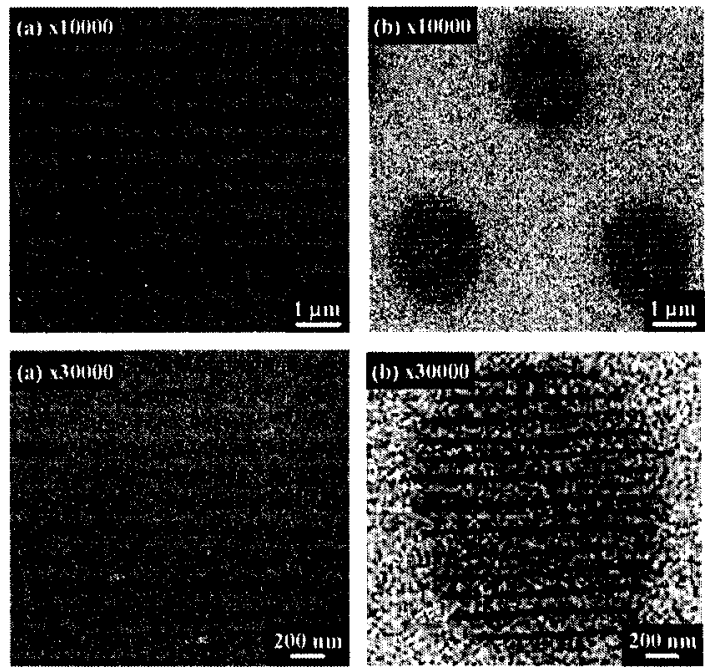

Fig.1 (a) Secondary electron images of silica glass surface polished close to the depth of focal spot. (b) Light "fingerprints": Backscattering electron images of the same surface. The magnification of the upper and lower images is $\mathrm{x} 10000$ and $\mathrm{x} 30000$ respectively. 
pulse energies of $1 \mu \mathrm{J}, 2 \mu \mathrm{J}$ and $2.8 \mu \mathrm{J}$ respectively and for the number of light pulses of $20 \times 10^{4}$ (Fig. 2a).

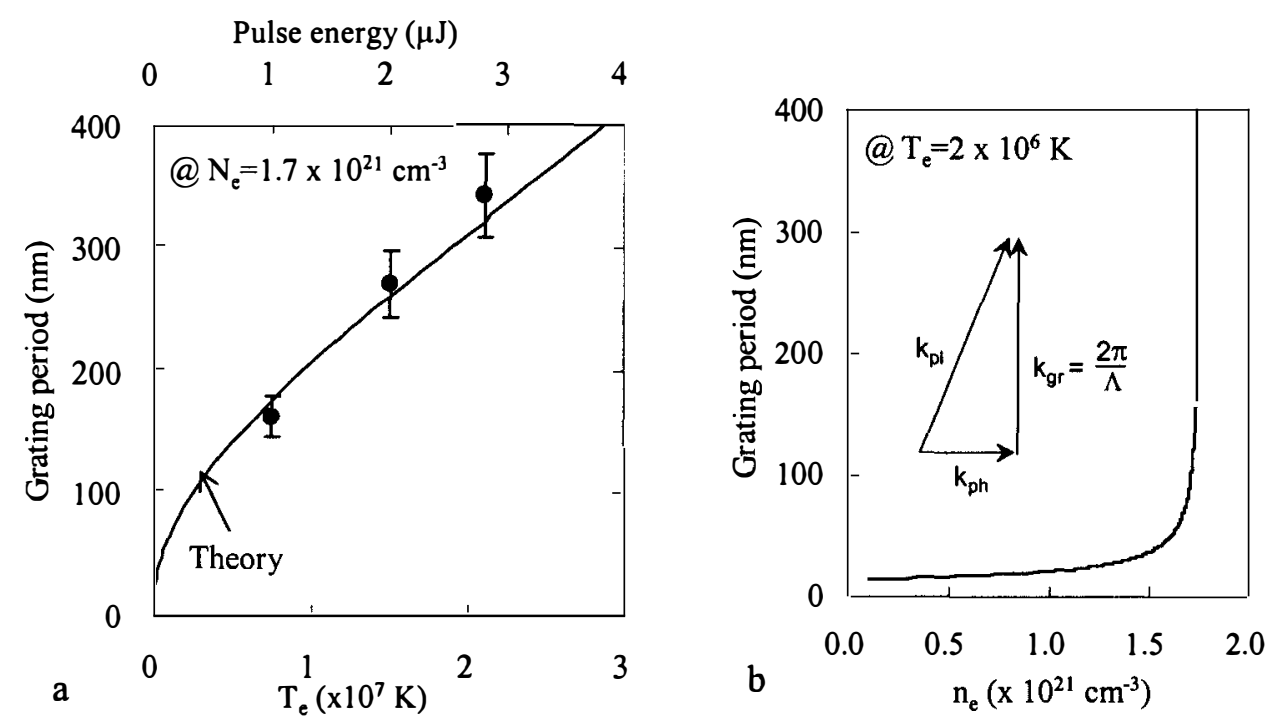

Fig.2 Theoretical dependence of self-organized grating period on electron temperature for $n_{e}=1.7 \times 10^{21} \mathrm{~cm}^{-3}$ (a) and electron concentration for $T_{e}=2 \times 10^{6} \mathrm{~K}$ (b). Experimental grating periods versus pulse energy are also shown (a). Insert is a wave vector phase-matching diagram (b).

The following explanation of the observed phenomenon is proposed. The light absorption in the electron plasma will excite bulk electron acoustic waves. These are longitudinal waves with the electric field component parallel to the direction of propagation. The coupling is increased by a periodic structure created via a pattern of interference between the incident light field and the electric field of the bulk electron acoustic wave, resulting in the periodic modulation of the electron plasma concentration and the structural changes in glass. The electron acoustic wave is generated only in the plane of light polarization and only in the direction defined by conservation of longitudinal component of the momentum (insert Fig. 2b). The latter condition is similar to the condition in Cherenkov's mechanism of nonlinear wave generation. Taking into account the energy conservation condition, the momentum conservation relation and the dispersion relation for Langmuir waves it is possible to calculate the dependences of the grating period on the electron temperature and the electron plasma density (Fig. $2 \mathrm{a}, \mathrm{b}$ ). Assuming that the electron temperature is proportional to the pulse energy due to one-photon absorption of light by the electron plasma, the experimental dependence of the grating period on the pulse energy is in very good agreement with the theoretical prediction (Fig. 2a). The interference between the light wave and the electron acoustic wave leads to modulation of the electron plasma concentration and the oxygen concentration.

Apart from the fundamental importance of the observed phenomenon as the first evidence of interference between light and electron acoustic waves, the observed light "fingerprints" are the smallest embedded structures ever created by light. The reported light-induced nanostructuring is a universal phenomenon in transparent materials and could be useful for optical recording and photonic crystal fabrication.

1. Z. Chang, A. Rundquist, H. Wang, M.M. Murnane, and H. Kapteyn, "Generation of coherent soft X Rays at $2.7 \mathrm{~nm}$ using high harmonics," Phys. Rev. Lett. 79, 2967-2970 (1997).

2. K. Miura, J. Qiu, H. Inouye, T. Mitsuyu, and K. Hirao, "Photowritten optical waveguides in various glasses with ultrashort pulse laser," Appl. Phys. Lett. 71, 3329-3331 (1997).

3. E. N. Glezer, and E. Mazur, "Ultrafast-laser driven micro-explosions in transparent materials," Appl. Phys. Lett. 71, 882-884 (1997).

4. H.-B. Sun, Y. Xu, K. Sun, S. Juodkazis, M. Watanabe, S. Matsuo, J. Nishii, and H. Misawa, "Arbitrarylattice photonic crystals created by multiphoton microfabrication," Opt. Lett. 26, 325-327 (2001).

5. B. C. Stuart, M. D. Feit, A. M. Rubenchik, B. W. Shore, and M.D. Perry, "Laser-induced damage in dielectrics with nanosecond to subpicosecond pulses," Phys. Rev. Lett. 74, 2248-2251 (1995).

6. D. Du, X. Liu, G. Korn, J. Squier, and G. Mourou, "Laser-induced breakdown by impact ionization in $\mathrm{SiO}_{2}$ with pulse widths from 7 ns to 150 fs," Appl. Phys. Lett. 64, 3071-3073 (1994).

7. S. R. L. Brueck and D. J. Ehrlich, "Stimulated surface plasma wave scattering and growth of a periodic structure in laser photodeposited metal films," Phys. Rev. Lett. 48, 1678-1681 (1982).

8. D. Homoelle, S. Wielandy, A.L. Gaeta, N. F. Borrelli, and C. Smith, "Infrared photosensitivity in silica glasses exposed to femtosecond laser pulses," Opt. Lett. 24, 1311-1313 (1999).

9. J. W.Chan, T. Huser, S. Risbud, and D.M. Krol, "Structural changes in fused silica after exposure to focused femtosecond laser pulses," Opt. Lett. 26, 1726-1728 (2001).

10. P. G. Kazansky, H. Inouye, T. Mitsuyu, K. Miura, J. Qiu, K. Hirao, and F. Starrost, "Anomalous anisotropic light scattering in Ge-doped silica glass," Phys. Rev. Lett. 82, 2199-2202 (1999).

11. J. D. Mills, P. G. Kazansky, E. Bricchi, and J. J. Baumberg, "Embedded anisotropic microreflectors by femtosecond-laser nanomachining," Appl. Phys. Lett., 81, 196-198 (2002).

12. L. Sudrie, M. Franco, B. Prade, and A. Mysyrowicz, "Writing of permanent birefringent microlayers in bulk fused silica with femtosecond laser pulses," Opt. Commun. 171, 279-284 (1999). 